\title{
NAPPA-Based Nanobiosensors for the Detection of Proteins and of Protein- Protein Interactions Relevant to Cancer
}

\author{
Nicola Luigi Bragazzi ${ }^{1,2}$, Rosanna Spera ${ }^{1,2}$, Eugenia Pechkova ${ }^{1,2}$ and Claudio Nicolini ${ }^{1,2,3, *}$ \\ ${ }^{1}$ Laboratories of Biophysics and Nanobiotechnology, Department of Experimental Medicine, University of Genoa, Italy \\ ${ }^{2}$ Nanoworld Institute Fondazione EL.B.A. Nicolini (FEN), Largo Redaelli 7, Pradalunga, Bergamo 24100, Italy \\ ${ }^{3}$ Virginia G. Piper Center for Personalized Diagnostics, Biodesign Institute, Arizona State University, USA \\ *Corresponding author: Claudio Nicolini, Nanoworld Institute Fondazione EL.B.A. Nicolini (FEN), Largo Redaelli 7, Pradalunga, Bergamo 24100, Italy, Tel: \\ +39-010-353-38217; Fax: +39-010-353-38215; E-Mail: info@fondazioneelba-nicolini.org
}

Received date: Novmber 6, 2013, Accepted date: March 22,2014, Published date: March 27, 2014

Copyright: (c) 2014 Bragazzi NL, et al. This is an open-access article distributed under the terms of the Creative Commons Attribution License, which permits unrestricted use, distribution, and reproduction in any medium, provided the original author and source are credited.

\begin{abstract}
In this manuscript, we report some proofs-of-principle and preliminary applications and results regarding the analytical quantification of protein expression of some genes biologically and clinically related to cancer. Experiments have been carried out coupling Nucleic Acid Programmable Protein Array (NAPPA) with a recently improved nanogravimetric apparatus which exploits the quartz crystal microbalance with frequency (QCM F) and quartz crystal microbalance with dissipation monitoring (QCM_D) technologies. The selected proteins are BRIP1, JUN and ATF2. Clinically relevant implications for cancer are envisaged and discussed, as well as future perspectives and developments.
\end{abstract}

Keywords: Cancer; Quartz Crystal Microbalance with Dissipation monitoring (QCM_D); Quartz Crystal Microbalance with Frequency monitoring (QCM_F); Nano-biosensors; Nucleic Acid Programmable Protein Array (NAPPA); Protein detection; Protein-protein interaction

\section{Introduction}

In the last years, the increasingly explosive evolution of the nanobiotechnologies applied to proteins, namely proteomics, both structural and functional, and specifically the development of more sophisticated protein arrays, has enabled scientists to investigate protein interactions and functions with an unforeseeable precision and wealth of details $[1,2]$. Novel proteins of unknown functions are the legacy of the post-genomics era [3] and protein arrays indeed make possible a high-troughput characterization and screening of thousands of interacting proteins, ranging from protein antibodies, proteinprotein, protein-ligand/protein-receptor, protein-drug, to enzymesubstrate screening and multianalyte medical diagnostic assays [4,5].

Advancements in protein science and in recombinant technologies, such as clone engineering, have directly linked DNA sequences and protein arrays, and since is more easier to store DNA and express it than obtaining purified proteins, this field of research has attracted a lot of interest from the scientific community [3]. Moreover, protein arrays [6-8] can be coupled with label-free approaches [9,10]: the socalled cell-free protein arrays, such as the Protein In Situ Array (PISA), the in situ puromycin-capture, the nano-well array format, and the DNA Array to Protein Array (DAPA). Label-free analysis do not require the use of any reporter elements (such as fluorescent, luminescent, radiometric, or colorimetric components), it can provide direct information on analyte kinetics and binding in the form of mass addition or depletion [10 and references therein,11].

In this manuscript, we report and discuss some preliminary results of protein expression of genes related to cancer. Experiments have been carried out coupling Nucleic Acid Programmable Protein Array (NAPPA) with a recently improved nanogravimetric apparatus which exploits the quartz crystal microbalance with frequency (QCM_F) and quartz crystal microbalance with dissipation monitoring (QCM_D) technologies. The selected proteins are BRIP1, JUN and ATF2 and their role and biological roles will be discussed further in this manuscript.

We chose NAPPA since this innovative technology avoids any time-consuming task in the difficult process of obtaining highly purified proteins, relying instead on the production of proteins from high quality super-coiled DNA [3]. For this purpose, complementary DNAs (cDNAs) of selected genes tagged with a C-terminal glutathione S-transferase (GST) are spotted on the microarray surface and expressed using a cell-free transcription/translation system (IVTT, in vitro transcription and translation). The newly expressed protein is captured on the array by an anti-GST antibody that have been coimmobilized with the expression clone on the microarray surface $[6,7]$.

The advantages and benefits of NAPPA technologies can be summarized in these highlights:

It spares time and the challenging process of obtaining highly purified proteins since expression, purification, stabilization and preservation of the protein and its subsequent spotting on the assay are replaced by a single step;

\section{Availability of cDNAs and clones;}

Protein integrity and stability, being freshly expressed in a cell-free mammalian system at the needed time, avoiding any not natural folding or post-translational modifications.

Proteins expressedon the NAPPA arrays are properly folded and biologically active. NAPPA microarray has, in fact, been successfully used for the study of different kinds of protein-protein interaction [6, and references therein] and more than $85 \%$ of the interactions that had been biochemically demonstrated and proved with purified proteins 
were detected on the array. Those interactions occur only between properly folded, non-denatured and bioactive proteins, demonstrating that the proteins on the array are properly functioning.

Despite the fact that HeLa cell lysate mix contains intrinsic GST [12], it has been verified that that the signal is coming from in situ expressed protein instead of intrinsic GST [13].

Our previous data taken together with data already extensively published in the literature show that proteins displayed on the array can be used for functional assays up to 24 hours after the protein expression. Moreover, NAPPA arrays printed with the cDNAs of interest can be even stored for more than six months. The expression of the proteins is performed just when the microarray is needed, and for this reason it is not necessary to worry about protein stability above the 24 hours window.

NAPPA microarrays can be useful in biomarkers discovery and for other clinical applications $[8,14]$, especially in the effort of moving towards Personalized Medicine [4,15].

NAPPA can be used also as a sensing system platform enabling the development of biosensor [10]. For this task we coupled NAPPA with a new generation of conductometric devices, namely QCM.

QCM_D indeed appears a promising tool to study protein-protein interactions especially in the field of oncology, both cellular and molecular. Some scholars have developed an acoustic QCM for studying the bio-mechanical and adhesion properties of tumor cells, namely epithelial cells (human embryonic kidney, HEK) 293T and cervical cancer (HeLa) and fibroblasts (African Green Monkey kidney cells, COS-7), deposited onto functionalized gold surfaces. They found QCM signature was unique for each kind of tumor cell line and even characterized the cell growth phase, enabling a precise and detailed identification [16]. Zhou was able to replicate these findings for mammary tumor, demonstrating a unique QCM fingerprint and a peculiar change in viscoelastic, dissipative and resistance properties of the cancer cells [17].

From a molecular point of view, Chen investigated the effects of EGF on cancer cells [18], while Prakrankamanant et al. implemented a biosensor for the sensing of Papillomavirus type 58 [19].

Chen and collaborators designed a QCM-based sensor for detecting ovarian cancer [20], while Bianco et al. exploited QCM_D for clinical uses, in order to early detect pancreatic ductal adenocarcinoma [21]. Wang and co-workers coupled label-free technologies with label approaches for the detection of acute leukemia [22]. Saint-Guirons focused instead on metastatic tumors [23].

QCM has proved to be a valuable tool to study the kinetics of antiblastic drugs, namely the signal transducer and activator of transcription 3 (STAT3) oncogene inhibitors [24]. Shang studied the structure of herceptin with QCM [25], its binding kinetics and affinity. Another intriguing pharmaceutical application of QCM is given by the dynamic measurements carried out by Zhou, that show that ginsenoside $\mathrm{Rh}(2)$ (G-Rh(2)) could inhibit the proliferation of adriamycin-resistant human breast cancer MCF-7 cells (MCF-7/ADR) in a concentration-dependent way [26].

In conclusion, QCM has been used for drugs investigation and for the study of biomarkers [27-30], alone and/or with other nanobiotechnologies, such as SPR (Surface Plasmon Resonance) and nanoparticles or aptamer-conjugated magnetic bead [31-33].
To the best of our knowledge, we coupled for the first time QCM_D with NAPPA technology for biomedical applications.

The objective of the present research regards the analysis of multiple protein-protein interaction towards potentially useful clinical applications, namely in the field of cancer studies.

\section{Experimental Section}

\section{QCM_D Conductometer}

Nanogravimetry makes use of functionalized piezoelectric quartz crystals (QC), which vary their resonance frequency (f) when a mass (m) is adsorbed to or desorbed from their surface. This is well described by the Sauerbrey's equation:

$$
\Delta \mathrm{f} / \mathrm{f} 0=-\mathrm{m} / \mathrm{A} \rho \mathrm{l}
$$

where $\mathrm{f} 0$ is the fundamental frequency, $\mathrm{A}$ is the surface area covered by the adsorbed molecule and $\rho$ and 1 are the quartz density and thickness, respectively.

Quartz resonators response strictly depends on the biophysical properties of the analyte, such as the viscoelastic coefficient. The dissipation factor (D) of the crystal's oscillation is correlated with the softness of the studied material and its measurement can be computed by taking into account the bandwidth of the conductance curve $2 \Gamma$, according to the following equation:

$$
\mathrm{D}=2 \Gamma / \mathrm{f}
$$

where $\mathrm{f}$ is the peak frequency value [ 10 and references therein].

In our analysis we introduced also a "normalized D factor", DN, that we defined as the ratio between the half-width half-maximum $(\Gamma)$ and the half value of the maximum value of the conductance (Gmax) of the measured conductance curves [10]:

$$
\mathrm{DN}=2 \Gamma / \mathrm{Gmax}
$$

$\mathrm{DN}$, is more strictly related to the curve shape, reflecting the conductance variation.

\section{NAPPA Experiments}

The QC-NAPPA arrays were printed at the Biodesign Institute, Arizona State University (Tempe, AZ USA) with 100 spots per QC, to enhance their sensitivity (in the previous experiments we utilized QC with a density of only 16 spots). For gene expression and protein production we employed a human lysate as IVTT system. Moreover the QCM_D software has been improved enabling the acquisition of the conductance curves at higher resolution [10].

We selected BRIP1, JUN and ATF2 for their clinical relevance.FANCJ/BRIP1/BACH1 (Fanconi anemia complementation group J protein/BRCA1-interacting protein 1/BRCA1-associated Cterminal helicase 1) is a 1249 amminoacids protein involved in breast, cervical and ovarian cancers, as well as in Fanconi anemia, which is a genetic cause of anemia, leading to acute myelogenous leukemia or to bone marrow failure. It is a DNA-dependent ATPase and helicase of the family RecQ DEAH, which interacts with the BRCT repeats domain of BRCA1. As helicase, it has an important role in preserving the chromatin structure and in facilitating and driving the replication process. It is encoded by the locus $17 \mathrm{q} 22-\mathrm{q} 24$, which is 184 kilobases long and has 19 exons [34], and is situated near to the locus encoding the BRCA1 protein. 
JUN encodes the c-Jun protein, which is a well-known oncogene, being the putative transforming gene of avian sarcoma virus 17 . It encodes indeed a protein with a strong similarity to the viral protein. It is encoded by the locus $1 \mathrm{p} 32-\mathrm{p} 31$ and its alteration leads to several malignancies [36].

ATF2 gene (chromosome 2q32) encodes a 505 amino-acids long transcription factor, that belongs to the leucine zipper family of DNAbinding proteins. Also ATF2 is an important component of signaling pathways whose alterations are a cause of malignant transformation $[36,37]$.

The viscoelastic properties of the NAPPA quartzes during binding process were monitored by a QCM_D instrument jointly developed with Elbatech srl (Marciana - LI, Italy). The quartz is connected to an RF gain-phase detector (Analog Devices, Inc., Norwood, MA, USA) and is driven by a precision Direct Digital Synthesizer (DDS) (Analog Devices, Inc., Norwood, MA, USA) around its resonance frequency, thus acquiring a conductance versus frequency curve ("conductance curve") which shows a typical Gaussian behaviour. The conductance curve peak is at the actual resonance frequency while the shape of the curve indicates how damped is the oscillation, i.e. how the viscoelastic effects of the surrounding layers affect the oscillation. The QCM_D software acquired simultaneously the frequency and dissipation factor variation versus time. Moreover, the software measures also the "normalized D factor", DN (for further information, the reader is referred to [10]).

In order to have a stable control of the temperature, the experiments were conduced in a temperature chamber. Microarrays were produced on standard nanogravimetry quartz used as highly sensitive transducers. The QC expressing proteins consists of $9.5 \mathrm{MHz}$, AT-cut quartz crystal of $14 \mathrm{~mm}$ blank diameter and $7.5 \mathrm{~mm}$ electrode diameter, produced by ICM (Oklahoma City, USA). The electrode material is $100 \AA \mathrm{Cr}$ and $1000 \AA \mathrm{Au}$ and the quartz is embedded into glass-like structures for easy handling. Each quartz was functionalized and printed with 100 NAPPA spots. The microarray production was performed at the Virginia G. Piper Center for Personalized Diagnostics, the Biodesign Institute at Arizona State University (ASU), Tempe, Arizona, USA. Quartzes gold surfaces were functionalized and coated with cysteamine to allow the immobilization of the NAPPA printing mix. Briefly, quartzes were washed three times with ethanol, dried with Argon and incubated over night at $4^{\circ} \mathrm{C}$ with $2 \mathrm{mM}$ cysteamine. Quartzes were then washed three times with ethanol in order to remove any unbound cysteamine and dried with Argon. DNA plasmids expressing GST tagged proteins were transformed into E. coli and DNA were purified using the NucleoPrepII anion exchange resin (Macherey Nagel). NAPPA printing mix was prepared with $1.4 \mu \mathrm{g} / \mathrm{ul}$ DNA, $3.75 \mu \mathrm{g} / \mathrm{ul} \mathrm{BSA} \mathrm{(Sigma),} \mathrm{5mM} \mathrm{BS3} \mathrm{(Pierce)} \mathrm{and} 66.5 \mu \mathrm{g}$ polyclonal capture GST antibody (GE Healthcares). Negative controls, named master mix (MM), were obtained replacing DNA for water in the printing mix. Samples were incubated at room temperature for 1 hour with agitation and then printed on the cysteamine-coated gold quartz using the Qarray II from Genetix. In order to enhance the sensitivity, each quartz was printed with 100 identical features of 300 microns diameter each, spaced by 350 microns center-to-center. There were immobilized different genes from DNASU plasmid repository, http://dnasu.asu.edu. Gene expression was performed in the laboratories of the Department of Experimental Medicine (DIMES), University of Genoa, immediately before the assay. Briefly, in vitro transcription and translation were performed using HeLa lysate mix (1-Step Human Coupled IVTT Kit, Thermo Fisher Scientific Inc.), prepared according to the manufacturers' instructions. The quartz, connected to the nanogravimeter inside the incubator, was incubated for $1.5 \mathrm{hr}$ at $30^{\circ} \mathrm{C}$ with $40 \mu \mathrm{l}$ of HeLa lysate mix for proteins synthesis and then, the temperature was decreased to $15^{\circ} \mathrm{C}$ for a period of 30 min to facilitate the proteins binding on the capture antibody (antiGST). After the processes of protein expression and capture, the quartz was removed from the instrument and washed at room temperature, in phosphate buffered saline (PBS) for 3 times. The quartz was then placed in the flux chamber for the protein-protein interaction analysis. The protocol described above was followed identically for both control QC (the one with only master mix (MM), i.e, all the NAPPA chemistry except the cDNA) and protein expressing QC.

The sensitivity of our NAPPA based biosensor was previously determined by the QC characteristics and the sensitivity of the nanogravimeter. At the moment, the minimum frequency shift detectable is of $0.05 \mathrm{~Hz}$ that corresponds to about $0.3 \mathrm{ng}$ of detected molecules (the reader is referred to [10]). Comparing our sensitivity data with the data available in the literature, QC characteristics appear in the range of other well-established techniques and procedures, such as SPR.

In our previous proofs-of-principle (described in more details in [38], to which the reader is referred), we conducted fluorescence experiments in parallel, experiments that enabled us to validate the QCM_D results, sensitivity and selectivity. QCM_D results have been calibrated up to the nanogram level, monitoring up to $1 \mathrm{Hertz}$ variation in frequency.

Moreover, QCM_D technique measures the viscosity of the expressed proteins linked directly to the structure and superfolding of the given protein, providing the scholars precious biophysical and biochemical information relevant to the protein structure and conformation. These details indeed allowe us to discriminate among different proteins of the same quantity being expressed in the same spot where different genes have been immobilized, a task that is a challenge even with fluorescence and labeled techniques. In $[10,38]$ we demonstrated that Jun, CdK2 and p53 could be discriminated in terms of the D-factor.

Reproducibility of the experiments was assessed computing the coefficient of variation $\left(\mathrm{CV}\right.$, or $\left.\sigma^{\star}\right)$, using the following equation (4):

$$
\sigma^{*}=\sigma / \mu(4)
$$

where $\sigma$ is the standard deviation, and $\mu$ is the mean.

The total amount of the expressed protein was computed in grams, being the usual unit of measurement in nanogravimetry, and later converted to molar concentration.

\section{Results and Discussion}

We analyzed the conductance curves acquired in NAPPA-QCs in different steps of the expressing and capturing process. Figures 1 to 2 show the conductance curves for NAPPA-QCs expressing BRIP1 and JUN (before and after ATF2 addiction). The curves were acquired both before the expression of NAPPA and after the protein expression/capture and washing process. The curves have been centered to their maximum frequency to better visualize the changing in bandwidths and conductance. These data pointed to a unique conductance curve shape for each protein and suggested the possibility to identify the expressed proteins by QCM-D even when combined on 
Page 4 of 7

the same expressing QC (as can bee sen in Figure 2). The conductance curves were acquired in different steps of NAPPA expression process.

In Tables 1 and 2 are reported the values of peak frequency (f), halfwidth half-maximum $(\Gamma)$ and the maximum value of the conductance
(Gmax) of the obtained conductance curves of Figure 1. There are reported also the $\mathrm{D}$ factor values $(\mathrm{D})$ and the values of the normalized $\mathrm{D}$ factor, $\mathrm{DN}=2 \Gamma / \mathrm{Gmax}$.

\begin{tabular}{|l|l|l|l|l|l|l|}
\hline BRIP1 conductance curves & $\mathrm{CV}$ & $f(\mathrm{~Hz})$ & $\Gamma(\mathrm{Hz})$ & $G \max \mathrm{mS})$ & $D^{*} 10^{3}$ & $\mathrm{DN}(\mathrm{Hz} / \mathrm{mS})$ \\
\hline IVTT addition(5 minutes) & $4.8 \%$ & 9466204 & 5709 & 0,47 & 1,21 & 24322,25 \\
\hline 10 minutes IVTT & $4.7 \%$ & 9466065 & 5720 & 0,47 & 1,21 & 24491,54 \\
\hline 15 minutes IVTT & $5.0 \%$ & 9465877 & 5480 & 0,46 & 1,16 & 23938 \\
\hline Post-capture & $5.2 \%$ & 9465172 & 5484 & 0,41 & 1,16 & 26751,21 \\
\hline Post-wash & $5.5 \%$ & 9463509 & 4637,75 & 0,25 & 0,98 & 37102 \\
\hline
\end{tabular}

Table 1: Main parameters of BRIP1 conductance curves, being collected at different steps of the NAPPA expression protocol ; ${ }^{*} \mathrm{CV}=\mathrm{Coefficient}$ of variation. We performed three independent experiments

We can notice an evident frequency decrease due to the human IVTT lysate addition and a change in viscoelastic properties of the quartzes after the human IVTT lysate addition, leading to a measurable increase of the bandwidth. During the incubation, on the contrary, the frequency and bandwidth variations were minimal. This effect could be related to two main effects: the first merely due to the IVTT lysate addition on the QC surface - when the QCM contacts with a solution, there is a decrement in frequency that is dependent upon the viscosity and the density of the solution, and there is a decrement in damping the resonant oscillation; the viscous loss is manifested as an increment in resistance of the QCM resonator; and the second due to the change of the composition of both QC surface and IVTT lysate after the gene expression and the protein synthesis and immobilization. The conductance curves acquired after PBS washing evidenced the further changes of solution in contact with the QC.

\begin{tabular}{|l|l|l|l|l|l|l|}
\hline BRIP1 conductance curves & $\Delta \mathrm{f}^{\prime}(\mathrm{Hz})$ & $\Delta \Gamma^{\prime}(\mathrm{Hz})$ & $\Delta \mathrm{G}^{\prime} \mathrm{max}(\mathrm{mS})$ & $\Delta \mathrm{D}^{\prime *} 10^{3}$ & $\Delta \mathrm{DN}(\mathrm{Hz} / \mathrm{mS})$ & $\mathrm{m}^{\prime}(\mu \mathrm{g})$ \\
\hline IVTT addition (10 minutes) & -139 & 11 & 0 & 0 & 169,29 & 0,6 \\
\hline IVTT addition (15 minutes) & -327 & -229 & $-0,01$ & $-0,05$ & $-384,25$ & 1,42 \\
\hline Post-capture & -1032 & -225 & $-0,06$ & $-0,05$ & 2428,96 & 4,48 \\
\hline Post-wash & -2695 & $-1071,25$ & $-0,22$ & $-0,23$ & 12779,75 & 1,42 \\
\hline
\end{tabular}

Table 2: Shift of the main parameters of BRIP1 conductance curves after lysate addition and relative amounts of protein immobilized onto the quartz surface. These calculations have been carried out in relation to the moments immediately after IVTT addition.

Using the calibration coefficient [10], we estimated the amount of molecules immobilized on the quartz surface at the end of immobilization protocol $(\mathrm{m})$.

To estimate the amount of proteins anchored on the QC surface after the NAPPA expression we had to take into account for the human IVTT lysate molecules nonspecifically adsorbed on the quartz surface. Assuming that on each QC surface there was the same specific adsorption, we could estimate it from reference quartz conductance curves. In particular, we considered the frequency shift between the reference QC conductance curves acquired immediately after the human IVTT lysate addition ("IVTT addition") and that acquired at the end of the protein anchorage ("90 min IVTT addition"); this value is $450 \mathrm{~Hz}$, that correspond to $2 \mu \mathrm{g}$ of molecules specifically adsorbed [10]. The values of immobilized BRIP1 molecules, therefore, was 2.28 $\mu \mathrm{g}$ while the amount of JUN was $1.69 \mu \mathrm{g}$. In terms of molar concentration, we found $0.02 \mathrm{nM}$ for BRIP1 (having a molar weight of $\sim 130 \mathrm{kDa}$ ) and $0.04 \mathrm{nM}$ for JUN (having a molar weight of $\sim 39 \mathrm{kDa}$ ).

\section{Conclusions}

In this manuscript, we have presented the results obtained applying our innovative conductometer, realized by combining NAPPA technology with QCM_D, to the characterization and analytical quantification of protein expression and protein-protein interactions in a multiparametric way, taking advantage of the multiple information provided by the analysis of the conductance curves (i.e. conductance, viscoelasticity and adsorbed mass).

The data here presented have been obtained employing a further improved version of our conductometer aimed to determine proteinprotein interactions; the NAPPA-QCs employed were printed with an higher number of spots (100), gene expression and protein synthesis was performed through a human lysate as IVTT system (to guarantee a higher protein yield) and also the QCM_D software has been updated and improved allowing the acquisition of the conductance curves at higher resolution. 


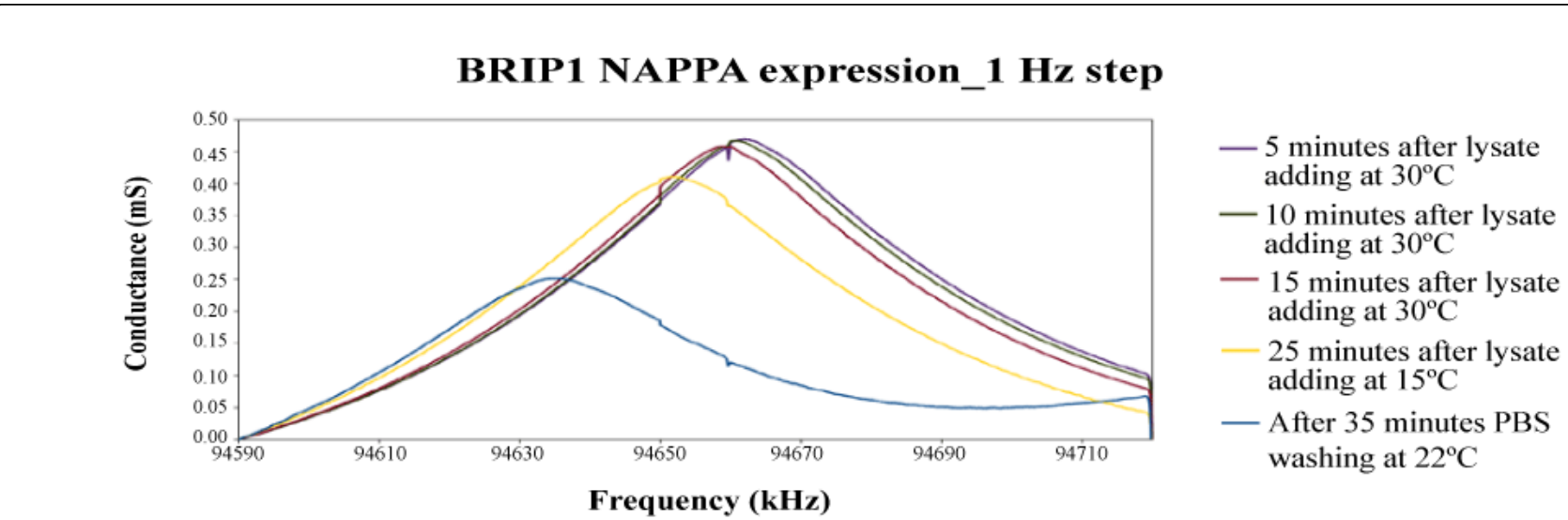

Figure 1: Conductance curves of BRIP1 QCs. The curves were collected in different steps of NAPPA expression protocol, as reported in the legend. The MM+BRIP1 conductance curves acquired with frequency acquisition steps of $1 \mathrm{~Hz}$.

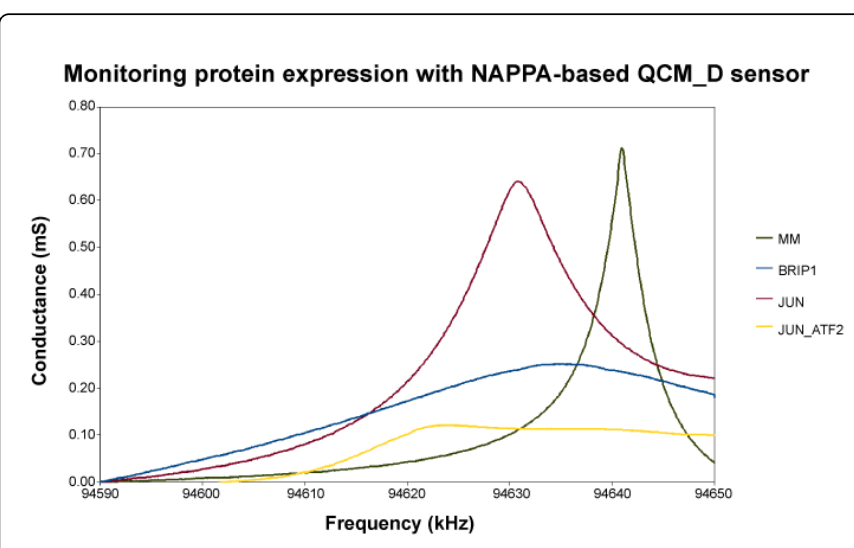

Figure 2: Conductance curves of different proteins and their negative control (MM, Master Mix). NAPPA-based QCM_D appears able to monitor and discriminate the protein expression of different proteins.

The data here presented have been obtained employing a further improved version of our conductometer aimed to determine proteinprotein interactions; the NAPPA-QCs employed were printed with an higher number of spots (100), gene expression and protein synthesis was performed through a human lysate as IVTT system (to guarantee a higher protein yield) and also the QCM_D software has been updated and improved allowing the acquisition of the conductance curves at higher resolution.

The main objective of this communication was to establish some proofs of principle by choosing proteins such as BRIP1, Jun and ATF2. A first interesting implication for potential clinical applications concerned the possibility to drastically reduce the time of protein expression and capture under our experimental conditions (this is true especially for BRIP1). Acquiring conductance curves each 5 minutes we noticed that after the first 15 minutes after IVTT lysate addition at $30^{\circ} \mathrm{C}$ the position and shape of the curves did not change anymore, likewise after few minutes at $15^{\circ} \mathrm{C}$, for protein capture, position and shape of the curves did not change anymore. We deduced from these results that the protein expression took place in the first minutes and that also their capture needed only few minutes and we performed experiments reducing the expression time from 90 minutes to 15 (at $30^{\circ} \mathrm{C}$ ) and the capture time from 30 to 5 minutes (at $\left.15^{\circ} \mathrm{C}\right)$. The results presented confirmed our hypothesis. The conductance curves obtained showed that protein expression and capture and protein-protein interactions were successfully performed. Applying the calibration coefficients, we were able to estimate the amount of protein immobilized on the biosensor surface. To estimate the amount of molecules aspecifically captured on the QC surface after the NAPPA expression (human IVTT lysate molecules not specifically adsorbed on the QC surface) we employed a reference QC (MM QC) and we estimated an amount of $2 \mu \mathrm{g}$ of molecules aspecifically adsorbed. For BRIP1 quartz we obtained $2.28 \mu \mathrm{g}$ (that is to say, a concentration of $0.02 \mathrm{nM}$ ), while for JUN quartz the amount was $1.69 \mu \mathrm{g}$ (corresponding to $0.04 \mathrm{nM}$ ).

As stated by Guerrini and collaborators [39], until today very few quantitative data about c-Jun expressions (and even less for BRIP1) are present in the literature, which presents only relative changes in magnitude orders during the carcinogenesis. However, taking the data together and assuming plausible biological perspective, c-Jun and BRIP1 expression vary from low nanomolar (as in our case) to micromolar range. Further studies, utilizing different cell lines or cellular extracts, as well as different plasmids are needed to replicate our findings.

The conductance curves of the different proteins and their negative control (MM, Master Mix) are summarized in Figure 2, where the NAPPA-based QCM_D biosensor appears able to monitor and discriminate the protein expression of the different selected proteins.

The multi-spot feature of our sensor could be further exploited for investigating the multi-protein expression and protein-protein interactions, becoming more clinically relevant. In fact, not single biomarkers are able to capture the diagnosis and prognosis of cancer, but a composite panel of proteins that could be properly detected via high-throughput sensors.

Future perspectives of this biosensor regard its clinical introduction for assessing cancer prognosis and make a personalized diagnosis and/or to deliver an individualized treatment. In particular, different mutations of the selected genes will be evaluated in terms of protein instability, functioning, and clinical implications for cancer prognosis. 


\section{Acknowledgments}

This project was supported by grants to FEN (Fondazione Elba Nicolini) and to Professor Claudio Nicolini of the University of Genova, Italy by the FIRB Nanobiosensors (ITALNANONET RBPR05JH2P_003) and by a grant Funzionamento from MIUR (Ministero dell'Istruzione, Università e Ricerca; Italian Ministry for Research and University).

The authors are very grateful to Prof. Joshua LaBaer for his precious cooperation and to Dr. Fernanda Festa for having printed the NAPPA at the Virginia G. Piper Center for Personalized Diagnostics, Biodesign Institute, Arizona State University, Tempe, AZ. USA

\section{Conflict of Interest}

The authors declare no conflict of interest.

\section{References}

1. Weston AD, Hood L (2004) Systems biology, proteomics, and the future of health care: toward predictive, preventative, and personalized medicine. J Proteome Res 3: 179-196.

2. Nicolini C, Adami M, Sartore M, Bragazzi NL, Bavastrello V, et al. (2012) Prototypes of newly conceived inorganic and biological sensors for health and environmental applications. Sensors (Basel) 12: 17112-17127.

3. Nicolini C, LaBaer, J (2010) Nanotechnology applications of Nucleic Acid Programmable Protein Arrays. In Functional Proteomics and Nanotechnology-based Microarrays ,Pan Stanford Series on Nanobiotechnology, Volume 2, Chapter 1, 1-29.

4. Nicolini C, Bragazzi N, Pechkova E (2012) Nanoproteomics enabling personalized nanomedicine. Adv Drug Deliv Rev 64: 1522-1531.

5. Tomizaki KY, Usui K, Mihara H (2010) Protein-protein interactions and selection: array-based techniques for screening disease-associated biomarkers in predictive/early diagnosis. FEBS J 277: 1996-2005.

6. Ramachandran N, Hainsworth E, Bhullar B, Eisenstein S, Rosen B, et al. (2004) Self-assembling protein microarrays. Science 305: 86-90.

7. Ramachandran N, Raphael JV, Hainsworth E, Demirkan G, Fuentes MG, et al. (2008) Next-generation high-density self-assembling functional protein arrays. Nat Methods 5: 535-538.

8. Gonzalez-Gonzalez M, Jara-Acevedo R, Matarraz S, Jara-Acevedo M, Paradinas S, et al. (2012) Nanotechniques in proteomics: protein microarrays and novel detection platforms. Eur J Pharm Sci 45: 499-506.

9. Adami M, Sallam S, Eggenhoffner R,. Sartore M, Hainsworth E, LaBaer J Nicolini C (2010) Label-free NAPPA via nanogravimetry. In Functional Proteomics and Nanotechnology-based Microarrays (Eds. Nicolini, C.; LaBaer, J.) Pan Stanford Series on Nanobiotechnology,Volume 2, Chapter 4, 95-108, London

10. Spera R, Bezerra Correia T T, Nicolini C (2013) NAPPA based nanogravimetric biosensor: Preliminary characterization. Sensors and Actuators B: Chemical: 682-688.

11. Rodahl M, Kasemo B (1996) Frequency and dissipation-factor responses to localized liquid deposits on a QCM electrode. Sensors and Actuators B: Chemical: 111-116.

12. Stergachis AB, MacLean B, Lee K, Stamatoyannopoulos JA, MacCoss MJ (2011) Rapid empirical discovery of optimal peptides for targeted proteomics. Nat Methods: 1041-1043.

13. Festa F, Rollins SM, Vattem K, Hathaway M, Lorenz P, et al. (2013) Robust microarray production of freshly expressed proteins in a human milieu. Proteomics Clin Appl: 372-377.

14. Dasilva N, Díez P, Matarraz S, González-González M, Paradinas S, et al (2012) Biomarker discovery by novel sensors based on nanoproteomics approaches. Sensors (Basel): 2284-2308.

15. Yu X, Schneiderhan-Marra N, Joos TO (2010) Protein microarrays for personalized medicine. Clin Chem : 376-387.
16. Da-Silva AC, Rodrigues R, Rosa LF, de-Carvalho J, Tomé B, et al. (2012) Acoustic detection of cell adhesion on a quartz crystal microbalance. Biotechnol Appl Biochem: 411-419.

17. Zhou T, Marx KA, Dewilde AH, McIntosh D, Braunhut SJ (2012) Dynamic cell adhesion and viscoelastic signatures distinguish normal from malignant human mammary cells using quartz crystal microbalance. Anal Biochem: 164-171.

18. Chen JY, Shahid A, Garcia MP, Penn LS, Xi J (2012) Dissipation monitoring for assessing EGF-induced changes of cell adhesion. Biosens Bioelectron: 375-381.

19. Prakrankamanant $\mathrm{P}$, Leelayuwat C, Promptmas C, Limpaiboon T, Wanram S, et al. (2013) The development of DNA-based quartz crystal microbalance integrated with isothermal DNA amplification system for human papillomavirus type 58 detection. Biosens Bioelectron: 252-257.

20. Chen Y, Huang XH, Shi HS, Mu B, Lv Q (2012) Rapid detection of ovarian cancer from immunized serum using a quartz crystal microbalance immunosensor. Asian Pac J Cancer Prev: 3423-3426.

21. Bianco M, Aloisi, A, Arima V,Capello M, Ferri-Borgogno S, et al.(2013) Quartz crystal microbalance with dissipation (QCM_D) as tool to exploit antigen-antibody interactions in pancreatic ductal adenocarcinoma detection. Biosens Bioelectron: 646-652.

22. Wang H, Zeng H, Shen G, Yu R (2006) Immunophenotyping of acute leukemias using a quartz crystal microbalance and monoclonal antibodycoated magnetic microspheres. Anal Chem: 2571-2578.

23. Saint-Guirons J, Ingemarsson B (2012) Using a quartz crystal microbalance biosensor for the study of metastasis markers on intact cells. Methods Mol Biol: 175-183.

24. Shi ZB, Zhao D, Huang YY, Du Y, Cao XR, et al. (2012) Discovery, synthesis, and evaluation of small-molecule signal transducer and activator of transcription 3 inhibitors. Chem Pharm Bull (Tokyo) 60: 1574-1580.

25. ShangY, Mernaugh R, Zeng X (2012) Characterization of the native and denatured herceptin by enzyme linked immunosorbent assay and quartz crystal microbalance using a high-affinity single chain fragment variable recombinant antibody. Anal Chem: 8164-8170.

26. Zhou B, Xiao X, Xu L, Zhu L, Tan L, et al. (2012) A dynamic study on reversal of multidrug resistance by ginsenoside Rhâ,, in adriamycinresistant human breast cancer MCF-7 cells. Talanta 88: 345-351.

27. Garai-Ibabe G, Grinyte R, Golub EI, Canaan A, de la Chapelle ML, et al. (2011) Label free and amplified detection of cancer marker EBNA-1 by DNA probe based biosensors. Biosens Bioelectron: 272-275.

28. MartÃnez-Rivas, A, Chinestra P, Favre G, Pinaud S, SÃverac C, et al. (2010) Detection of label-free cancer biomarkers using nickel nanoislands and quartz crystal microbalance. Int J Nanomedicine: 661-668.

29. Tarantola M, Marel AK, Sunnick E, Adam H, Wegener J, et al. (2010) Dynamics of human cancer cell lines monitored by electrical and acoustic fluctuation analysis. Integr Biol (Camb): 139-150.

30. Marx K A, Zhou T, Montrone A, McIntosh D, Braunhut S J (2007) A comparative study of the cytoskeleton binding drugs nocodazole and taxol with a mammalian cell quartz crystal microbalance biosensor: different dynamic responses and energy dissipation effects. Anal Biochem: 77-92.

31. UludaÄŸ Y,Tothill IE (2010) Development of a sensitive detection method of cancer biomarkers in human serum (75\%) using a quartz crystal microbalance sensor and nanoparticles amplification system. Talanta: 277-282.

32. Uludag Y, Tothill IE (2012) Cancer biomarker detection in serum samples using surface plasmon resonance and quartz crystal microbalance sensors with nanoparticle signal amplification. Anal Chem: 5898-5904.

33. Pan Y, Guo M, Nie Z, Huang Y, Pan, C, et al (2010)Selective collection and detection of leukemia cells on a magnet-quartz crystal microbalance system using aptamer-conjugated magnetic beads. Biosens Bioelectron: 1609-1614. 
Citation: Bragazzi NL, Spera R, Pechkova E, Nicolini C (2014) NAPPA-Based Nanobiosensors for the Detection of Proteins and of ProteinProtein Interactions Relevant to Cancer. J Carcinog \& Mutagen 5: 166. doi:http://dx.doi.org/10.4172/2157-2518.1000166

Page 7 of 7

34. Ma XD, Cai GQ, Zou W, Huang YH, Zhang JR, et al. (2013) First evidence for the contribution of the genetic variations of BRCA1interacting protein 1 (BRIP1) to the genetic susceptibility of cervical cancer. Gene 524: 208-213.

35. Meng Q, Xia Y (2011) c-Jun, at the crossroad of the signaling network. Protein Cell: $889-898$.

36. Gozdecka M, Breitwieser W (2012) The roles of ATF2 (activating transcription factor 2) in tumorigenesis. Biochem Soc Trans: 230-234.
37. Bhoumik A, Ronai Z (2008) ATF2: a transcription factor that elicits oncogenic or tumor suppressor activities. Cell Cycle: 2341-2345.

38. Spera R, Festa F, Bragazzi NL, Pechkova E, LaBaer J, et al. (2013) Conductometric monitoring of protein-protein interactions. J Proteome Res: 5535-5547.

39. Guerrini L, Pazos E, Penas C, Vázquez ME, Mascareñas JL, et al. (2013) Highly sensitive SERS quantification of the oncogenic protein c-Jun in cellular extracts. J Am Chem Soc: 10314-10317. 